\title{
Piezo1 channel activates ADAM10 sheddase to regulate Notch1 and gene expression
}

Vincenza Caolo $^{1 \dagger}$, Marjolaine Debant ${ }^{1 \dagger}$, Naima Endesh ${ }^{1}$, T Simon Futers ${ }^{1}$, Laeticia Lichtenstein ${ }^{1}$, Gregory Parsonage ${ }^{1}$, Elizabeth AV Jones ${ }^{2 *}$, David J Beech ${ }^{1 *}$

${ }^{1}$ Leeds Institute of Cardiovascular and Metabolic Medicine, School of Medicine, University of Leeds, Leeds, England, UK. ${ }^{2}$ Department of Cardiovascular Sciences, Centre for Molecular and Vascular Biology, KU Leuven, Belgium.

${ }^{\dagger}$ Equal contributors

*Authors for correspondence: Professor David J Beech, Leeds Institute of Cardiovascular and Metabolic Medicine, School of Medicine, LIGHT Building, Clarendon Way, University of Leeds, Leeds LS2 9JT, UK. Telephone +44 113343 4323. Email d.j.beech@leeds.ac.uk. Dr Elizabeth AV Jones, Department of Cardiovascular Sciences, Centre for Molecular and Vascular Biology, KU Leuven, Belgium. Telephone +32 163 45775. Email liz.jones@kuleuven.be. 


\begin{abstract}
Mechanical force has emerged as a determinant of Notch signalling but the mechanisms of force sensing and coupling to Notch are unclear. Here we propose a role for Piezol channels, the recently identified mechanosensors of mammalian systems. Piezo1 channel opening in response to shear stress or a chemical agonist led to activation of a disintegrin and metalloproteinase domain-containing protein 10 (ADAM10), a $\mathrm{Ca}^{2+}$-regulated transmembrane sheddase that mediates S2 Notch1 cleavage. Consistent with this observation there was increased Notch1 intracellular domain (NICD) that depended on ADAM10 and the downstream S3 cleavage enzyme, $\gamma$-secretase. Endothelial-specific disruption of Piezo1 in mice led to decreased Notch1-regulated gene expression in hepatic vasculature, consistent with prior evidence that Notch1 controls hepatic perfusion. The data suggest Piezo1 as a mechanism for coupling physiological force at the endothelium to ADAM10, Notch1, gene expression and vascular function.
\end{abstract}

\title{
Key Words
}

Ion channel; Notch signalling; Proteinase; Endothelial cell.

\section{Introduction}

Mammalian Notch proteins were identified following studies in D. melanogaster that linked genetic abnormality to wing notch ${ }^{1}$. Extensive research then revealed major roles in the transfer of information between cells in health and disease ${ }^{1}$. Each of the four Notch receptors (Notch1-4) is a membrane protein that is trans coupled to a membrane-anchored ligand such as Deltalike 4 (DLL4). Though the initiation of Notch signalling is often considered to occur through ligand-receptor complex formation, mechanical force also plays an important role in this activation whereby a pulling force arising from ligand endocytosis causes trans activation ${ }^{1,2}$. Furthermore it recently became apparent that frictional force from fluid flow also stimulates Notch1, but how this force couples to the Notch mechanism is unknown ${ }^{3-6}$. Therefore mechanical forces would seem to play key roles in Notch regulation. Further information is needed on how this is achieved.

Piezo1 channels are key players in the sensing of shear stress and lateral force applied to plasma membranes (membrane tension) ${ }^{7-14}$. While there are multiple candidate sensors, Piezo1 channels are notable because of broad agreement amongst investigators that they are direct sensors of physiological force. As such they are now considered to be bona fide force sensors rather than proteins that, whilst affected by force, do not appear to have evolved to sense and transduce force as a primary function. Piezo 1 channels are exquisitely sensitive to membrane tension ${ }^{15}$ and readily able to confer force-sensing capacity on cells that are otherwise poorly sensitive ${ }^{7,9}$. Reconstitution of Piezo1 channels in artificial lipid bilayers generates force-sensing channels ${ }^{16}$ and native Piezol channels in excised membrane patches respond robustly to mechanical force in the absence of intracellular factors ${ }^{10}$.

Piezo1 channels are $\mathrm{Ca}^{2+}$-permeable non-selective cationic channels, so when force causes them to open there is $\mathrm{Ca}^{2+}$ entry, elevation of the cytosolic $\mathrm{Ca}^{2+}$ concentration and regulation of $\mathrm{Ca}^{2+}$-dependent mechanisms ${ }^{7,8}$. Potentially relevant to such a system is $\mathrm{Ca}^{2+}$ and $\mathrm{Ca}^{2+}$-calmodulin regulation of ADAM10 ${ }^{17,18}$, a metalloprotease or sheddase that catalyses rate-limiting S2 cleavage of Notch1 prior to $\gamma$-secretase-mediated S3 cleavage and release of Notch1 intracellular domain (NICD), driving downstream transcription ${ }^{1,19,20}$. Therefore, we speculated about a potential relationship between Piezo1 and Notch1, using endothelial cells to investigate this idea because both proteins are prominent in these cells and have established functional significance in them ${ }^{1,8-10,12,19}$. Because mechanical force can affect numerous mechanisms, we explored the relationship by specifically activating Piezo1 channels with a synthetic small-molecule agonist (Yoda1) that acts directly to enhance force sensitivity ${ }^{21-24}$. Because there is inherent force in cell membranes and force arises through cell-cell and cell-substrate contact, Yoda1 can be used to activate the channels in endothelial cells without applying an exogenous force ${ }^{24}$ that might otherwise complicate the analysis by concurrently stimulating parallel mechanisms. Piezo1 dependence of Yoda1's actions was investigated and the effect of fluid flow was also determined. 


\section{Results}

Piezo1 regulates NICD abundance. To activate Piezo1 channels without stimulating other forcesensing mechanisms we applied $0.2 \mu \mathrm{M}$ Yoda1, the concentration required for half-maximal activation of native endothelial Piezo1 channels ${ }^{24}$. After 30-min Yoda1 treatment, human microvascular endothelial cells (HMVEC-Cs) showed 1.5-2 times as much NICD as controls (Figure 1a-d). Short interfering (si) RNA specific to Piezo1 (Supplementary Figure S1) reduced basal NICD to about half its control value and the Yodal effect was suppressed (Figure 1b). The data are consistent with the reported ability of shear stress to increase NICD ${ }^{4}$ and suggest importance of Piezo1 for basal Notch1 processing and ability of Yoda1 to stimulate production of NICD.

$\gamma$-secretase is required. If Piezo1 channels do indeed promote NICD release, the effect should depend on $\gamma$-secretase ${ }^{1}$. Therefore we tested the role of $\gamma$-secretase by treating cells with $10 \mu \mathrm{M}$ DAPT $(\mathrm{N}-[\mathrm{N}-$

a.

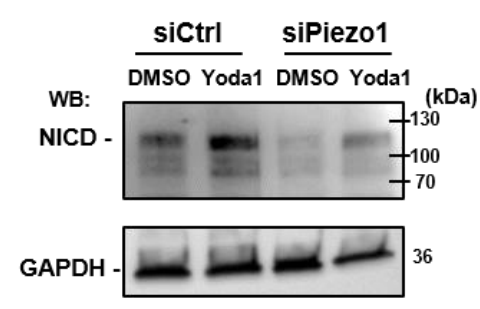

c.

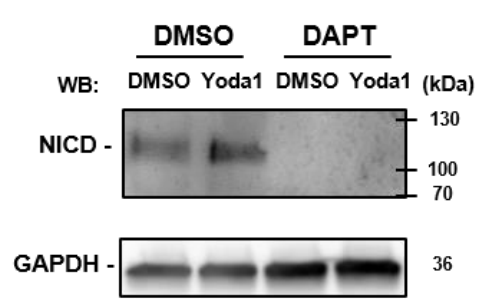

b.

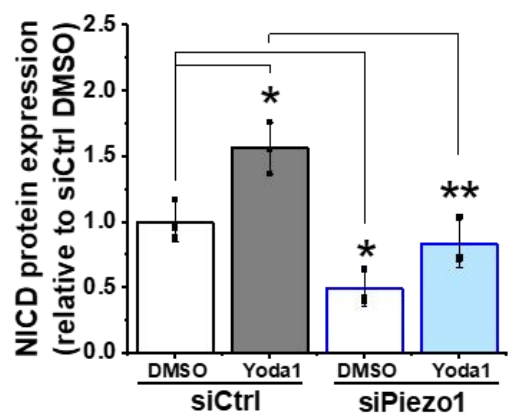

d.

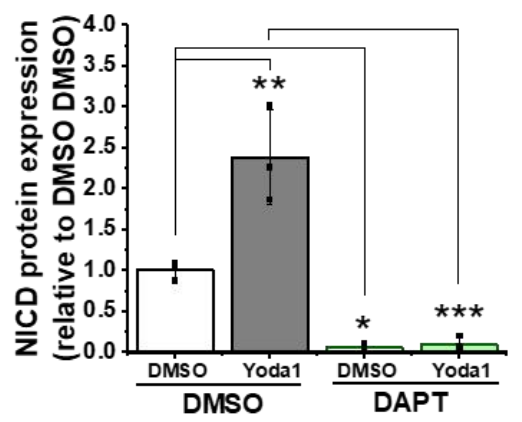

Figure 1: Piezo1 increases NICD abundance via $\gamma$-secretase. (a) Representative Western blot labelled with anti-NICD and anti-GAPDH (loading control) antibodies for HMVEC-Cs treated for $30 \mathrm{~min}$ with $0.2 \mu \mathrm{M}$ Yoda1 or vehicle (DMSO) after transfection with control siRNA (siCtrl) or Piezo1 siRNA (siPiezo1). The expected mass of NICD is $110 \mathrm{kDa}$. Lower molecular bands were also apparent in some experiments and may have been degraded NICD. (b) Quantification of data of the type exemplified in (a), showing mean \pm SD for abundance of NICD normalized to siCtrl DMSO $(n=3)$. (c) Representative Western blot labelled with anti-NICD and anti-GAPDH antibodies for HMVEC-Cs treated for 30 min with $0.2 \mu \mathrm{M}$ Yoda1 or vehicle (DMSO) in the absence or presence of $10 \mu \mathrm{M}$ DAPT. (d) Quantification of data of the type exemplified in (c), showing mean \pm SD data for abundance of NICD normalized to vehicle (DMSO) control $(n=3)$. Statistical analysis: Two-way ANOVA test was used, indicating $* \mathrm{P}<0.05, * * \mathrm{P}<0.01, * * * \mathrm{P}<0.001$.

(3,5-difluorophenacetyl)-1-alanyl]-S-phenylglycine t-butylester), a commonly used $\gamma$-secretase inhibitor $^{4,25}$. DAPT had an effect on NICD that was similar to that of Piezo1 siRNA, reducing basal NICD and ablating the ability of Yoda1 to increase NICD (Figure 1c, d). DAPT inhibited Yoda1-evoked $\mathrm{Ca}^{2+}$ entry by about $30 \%$, which was unlikely to have been sufficient to explain its effect on NICD (Supplementary Figure S1). The data suggest that Piezo1-mediated and constitutively generated NICD require $\gamma$-secretase. 
bioRxiv preprint doi: https://doi.org/10.1101/732370; this version posted August 12, 2019. The copyright holder for this preprint (which was not certified by peer review) is the author/funder, who has granted bioRxiv a license to display the preprint in perpetuity. It is made available under aCC-BY 4.0 International license.

ADAM10 is important. In order to understand how Piezo1 channels couple to Notch1 we next considered the role of ADAM10, which is required for $\mathrm{S} 2$ cleavage before $\gamma$-secretase can act ${ }^{1}$. SiRNA targeted to ADAM10 (Supplementary Figure S2) had an effect on NICD that was remarkably similar to that of Piezo1 siRNA and DAPT (Figure 2a, b cf Figure 1). It tended to reduce basal NICD, and Yoda1 no longer had significant effect (Figure 2b). The data suggest that ADAM10 is required for effects of Piezo1 on NICD.

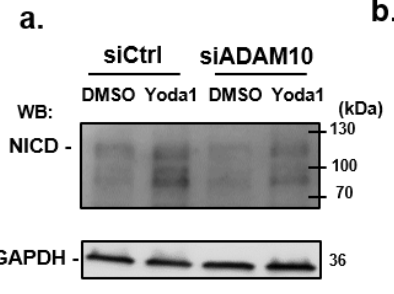

e.

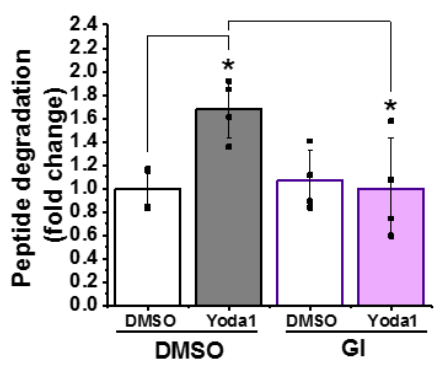

b.

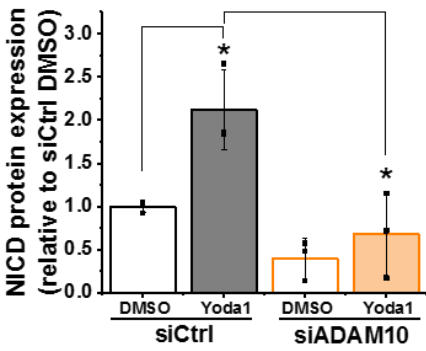

c.
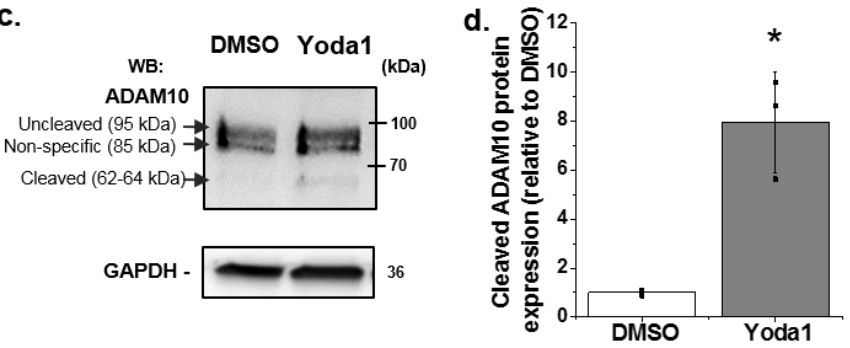

h.

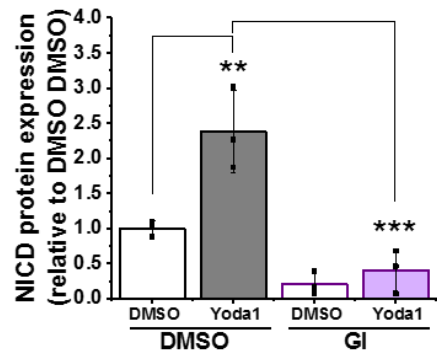

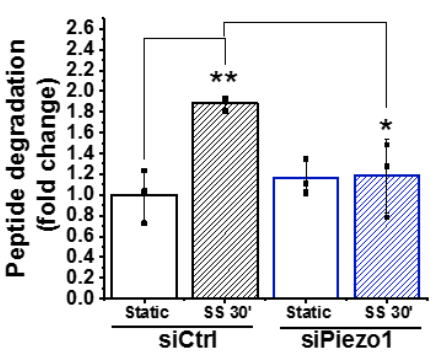

Figure 2: ADAM10 is important for Piezo1 regulation of NICD and activated by shear stress. (a) Representative Western blot labelled with anti-NICD and anti-GAPDH antibodies for HMVEC-Cs treated for 30 min with $0.2 \mu$ M Yoda1 or vehicle (DMSO) after transfection with control siRNA (siCtrl) or ADAM10 siRNA (siADAM10). (b) Quantification of data of the type exemplified in (a), showing mean \pm SD data for abundance of NICD normalized to siCtrl DMSO $(\mathrm{n}=3)$. $(\mathbf{c}, \mathbf{d})$ Quantification of uncleaved $(95 \mathrm{kDa})$ and cleaved (62-64 kDa) ADAM10 in HMVEC-Cs after treatment for 30 min with Yoda1 $(0.2 \mu \mathrm{M})$. The $85 \mathrm{kDa}$ band between the uncleaved and cleaved ADAM10 was non-specific labelling not related to ADAM10 (Supplementary Figure S2). Data represent mean \pm SD $(n=3)$ and normalization was to the reference protein, GAPDH. (e) ADAM10 enzyme activity assessed by specific peptide degradation and subsequent fluorescence emission after 30 min treatment of HMVEC-Cs with $0.2 \mu \mathrm{M}$ Yoda1 in the absence or presence of $5 \mu \mathrm{M}$ GI254023X (GI). Data are shown as mean \pm SD data $(n=4)$ relative to DMSO condition. (f) Example Western blot labelled with anti-NICD and anti-GAPDH antibodies for HMVEC-Cs treated for $30 \mathrm{~min}$ with $0.2 \mu \mathrm{M}$ Yoda1 or vehicle (DMSO) in the absence or presence of $5 \mu \mathrm{M}$ GI254023X (GI). (g) Quantification of data of the type exemplified in (f), showing mean \pm SD data for abundance of NICD normalized to DMSO $(\mathrm{n}=3)$. (h) ADAM10 enzyme activity as in (e) but after 30 min exposure of HMVEC-Cs to 10 dyn. $^{-}$ 2 laminar shear stress (SS). Static was without SS. Cells were transfected with control siRNA (siCtrl) or Piezo1 siRNA (siPiezo1). Data are shown as mean \pm SD data $(n=3)$ relative to static condition. Statistical analysis: Two-way ANOVA test was used for $(\mathbf{b}, \mathbf{e}, \mathbf{g}, \mathbf{h})$, indicating $* \mathrm{P}<0.05, * * \mathrm{P}<0.01$, *** $\mathrm{P}<0.001$; t-Test was used for $(\mathbf{d})$, indicating $* \mathrm{P}<0.05$, $* * * \mathrm{P}<$ 0.001 .

ADAM10 enzyme activity is needed. To investigate if ADAM10 activity mediates the effect of Yoda1, we first quantified the abundance of its cleaved form, a 62-64 $\mathrm{kDa}$ protein that is generated by proprotein convertase to enable enzymatic activity ${ }^{20}$. In HMVEC-Cs, the majority of ADAM10 was in the uncleaved form, a protein of $95 \mathrm{kDa}$ that is inactive (Figure 2c, Supplementary Figure S2). Yoda1 increased the abundance of the cleaved form, but the quantity remained small relative to the total protein (Figure 2c, d). To investigate if the ADAM10 was enzymatically active ${ }^{18}$ we used a cell-based activity assay. Yoda1 increased ADAM10 activity (Figure 2e). The effect was prevented by the widely used ADAM10 inhibitor GI254023X ((2R,3S)-3-(Formyl-hydroxyamino)-2-(3-phenyl-1-propyl) butanoic acid[(1S)-2,2-dimethyl-1-methylcarbamoyl-1-propyl] amide) which is thought to act via the catalytic $\operatorname{sit}^{26}$ (Figure 2e). GI254023X (5 $\left.\mu \mathrm{M}\right)$ affected NICD similarly to Piezo1 siRNA and ADAM10 siRNA, 
reducing both basal NICD and the ability of Yoda1 to increase NICD (Figure 2f, g). A 10-fold lower concentration of GI254023X (500 nM) inhibited the Yoda1 effect on NICD (Supplementary Figure S2), consistent with its nanomolar potency against ADAM10 $0^{26}$. GI254023X $(5 \mu \mathrm{M})$ had no effect on Yoda1evoked $\mathrm{Ca}^{2+}$ entry (Supplementary Figure S2). $\mathrm{Gd}^{3+}(30 \mu \mathrm{M})$, a blocker of the Piezo1 channel pore, inhibited the ability of Yoda1 to activate ADAM10, consistent with $\mathrm{Ca}^{2+}$ entry through the channel being required (Supplementary Figure S2). The data suggest that Piezo1-mediated stimulation of ADAM10 enzyme activity is necessary for basal and stimulated effects on Notch1 cleavage.

Shear stress activates ADAM10, via Piezo1. To further investigate ADAM10, we applied 10 dyn.cm ${ }^{2}$ laminar shear stress, a physiological stimulator of Piezo1 channels ${ }^{9}$. In response to this stimulus there was also increased ADAM10 enzymatic activity, which was Piezo1-dependent (Figure 2h). The data suggest that shear stress acts, like Yoda1, to stimulate ADAM10 via Piezo1 channels.

Function significance for downstream gene expression. To investigate if there is functional significance of Piezo1-regulated NICD, we quantified Notch1-regulated gene expression, focussing on the HES1 gene which is Notch1- and flow- regulated ${ }^{4}$ and DLLA, which is itself Notch1 regulated ${ }^{27}$. Yoda1 caused increased expression of both HESI and DLL4 genes (Figure 3), consistent with Yoda1's effect on NICD (Figure 1). The effects of Yoda1 were suppressed by Piezo1 siRNA (Figure 3a, b), DAPT (Figure 3c, d), ADAM10 siRNA (Figure 3e, f) and GI254023X (Figure 3g, h). In most cases, there was little or no change in constitutive gene expression, suggesting that it is regulated mostly by other mechanisms in these cultured cells (HMVEC-Cs). Nevertheless, the data suggest that Piezo1 signalling via Notch1 can be functionally important for downstream gene regulation.

a.

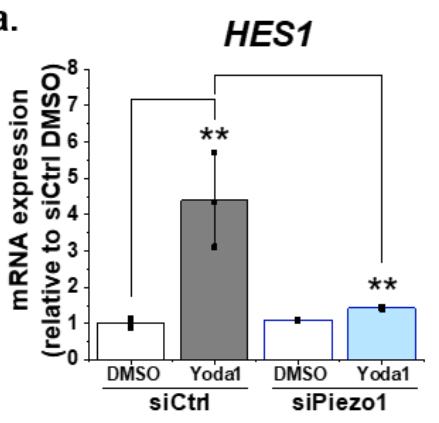

e.

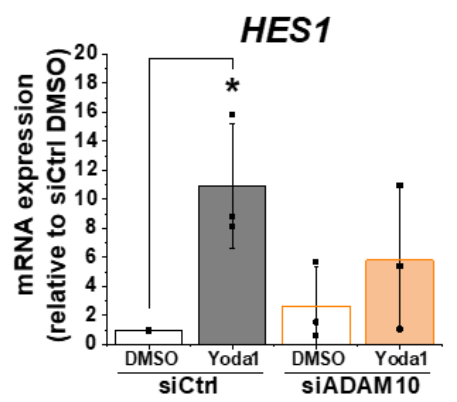

b.

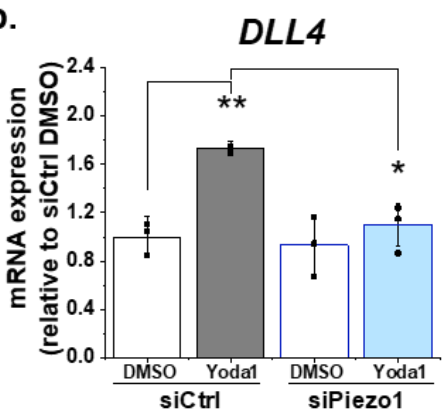

f.

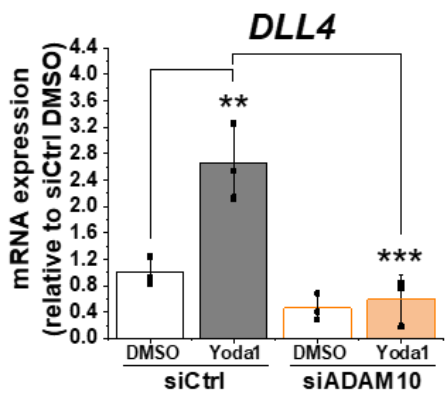

c.

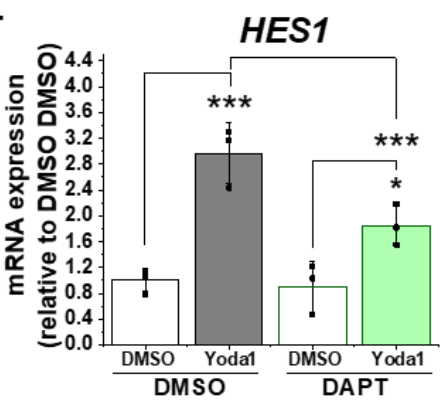

g.

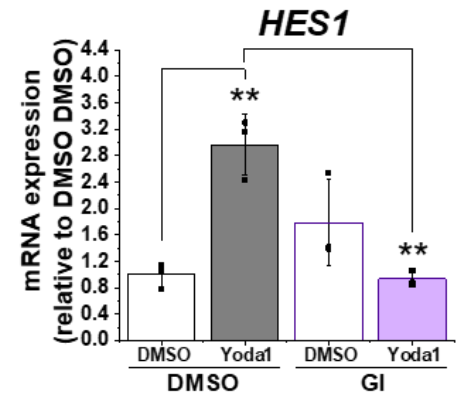

d.

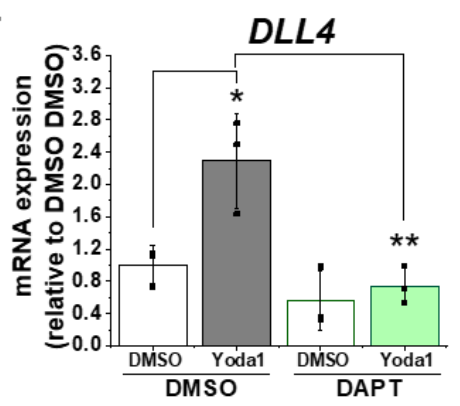

h.

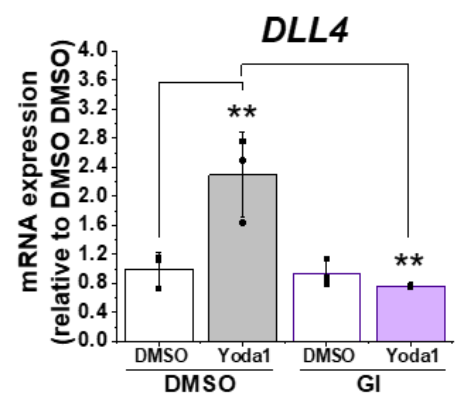

Figure 3: Function significance for downstream gene expression. $(\mathbf{a}, \mathbf{b})$ Summarized mean $\pm \mathrm{SD}(\mathrm{n}=3)$ quantitative PCR data for fold-change in HES1 (a) and DLL4 (b) mRNA in HMVEC-Cs treated for 30 min with $0.2 \mu$ M Yodal or vehicle (DMSO) after transfection with control siRNA (siCtrl) or Piezo1 siRNA (siPiezo1). (c, d) Summarized mean \pm SD $(n=3)$ quantitative PCR data for fold-change in HESI (c) and DLL4 (d) mRNA in HMVEC-Cs treated for 30 min with $0.2 \mu \mathrm{M}$ Yoda1 in the absence or presence of $10 \mu \mathrm{M}$ DAPT for $2 \mathrm{hr}$. (e, f) Summarized mean \pm SD $(\mathrm{n}=3)$ quantitative PCR data for foldchange in HES1 (e) and DLLA (f) mRNA in HMVEC-Cs treated for 30 min with $0.2 \mu \mathrm{M}$ Yoda1 or vehicle (DMSO) after transfection with control siRNA (siCtrl) or ADAM10 siRNA (siADAM10). (g, h) Summarized mean \pm SD ( $n=3$ ) quantitative PCR data for fold-change in HES1 (g) and DLL4 (h) mRNA in HMVEC-Cs treated for 30 min with $0.2 \mu \mathrm{M}$ Yoda1 in the absence or presence of $5 \mu \mathrm{M}$ GI254023X (GI) for $2 \mathrm{hr}$. Normalization and statistical analysis: mRNA expression was normalized to GAPDH mRNA abundance. Two-way ANOVA test was used, indicating $* \mathrm{P}<0.05, * * \mathrm{P}<0.01, * * * \mathrm{P}<0.001$. 
Importance for hepatic vasculature gene regulation. To test the physiological relevance, we investigated liver where functional microvascular Piezo1 channels have been detected at the adult stage $^{10}$ and where Notch1 is important for sinusoid development ${ }^{19,28}$. To specifically ask about endothelial biology, liver endothelial cells were acutely isolated after 2-weeks in vivo conditional Piezo1 disruption in endothelium of adult mice $\left(\right.$ Piezo $\left.^{\Delta \mathrm{ECC}}\right)$, as previously described ${ }^{10}$. Neither mice nor isolated cells received Yoda1, so Piezo1 channel activity should have arisen only due to physiological forces existing in vivo prior to cell isolation. Strikingly, expression of Hesl and Dll4 genes was downregulated in Piezo1 ${ }^{\triangle \mathrm{EC}}$ endothelial cells (Figure 4a, b) (Supplementary Figure S3). Heyl and EphrinB2, other genes known to be Notch1- and flow-regulated ${ }^{4,6}$, were also downregulated (Figure 4c, d). Expression of the reference gene, $\beta$-actin, and endothelial marker gene, Tie2, was not affected (Supplementary Figure S3). Whole liver showed no change or trends towards decreased expression of Hes1, Dll4, Heyl and Ephrin B2 in Piezo1 ${ }^{\triangle \mathrm{EC}}$ mice, consistent with effects restricted to the endothelial cell population (Supplementary Figure S4). The data suggest that endothelial Piezo1 normally positively regulates Notch1 signalling in hepatic vasculature.

a.

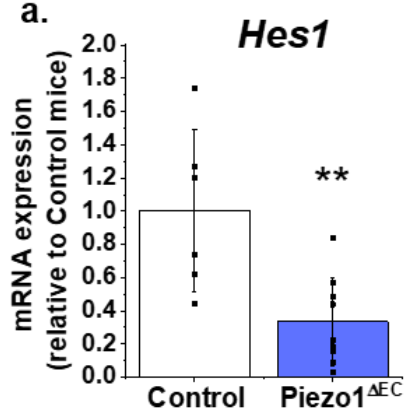

b.

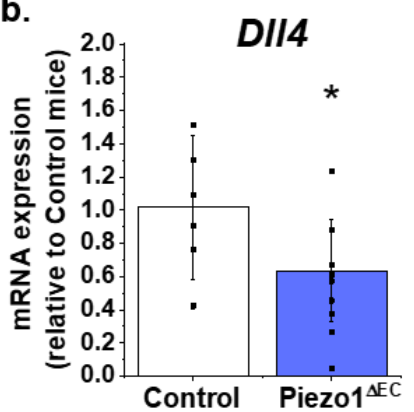

c.

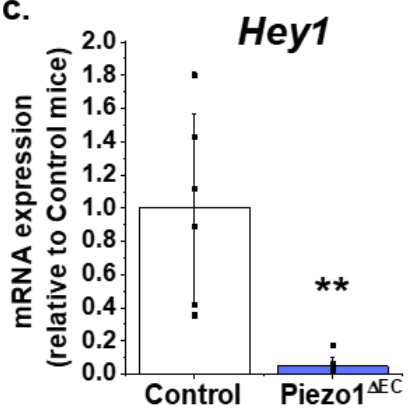

d.

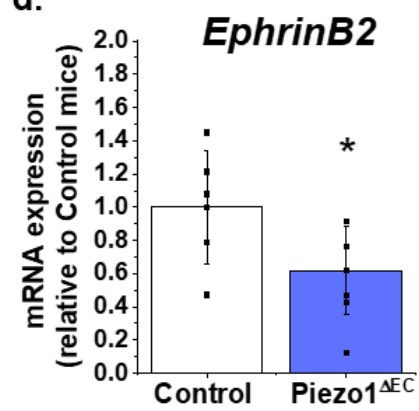

Figure 4: Endothelial Piezo1 promotes Notch1-regulated gene expression in liver. Hes1 (a), Dll4 (b), Heyl (c) and EphrinB2 (d) mRNA expression in liver endothelial cells freshly-isolated from control mice (Control, $\mathrm{n}=6)$ and endothelial Piezo1 knockout mice $\left(\right.$ Piezo1 $\left.^{\triangle \mathrm{EC}}\right)(\mathrm{n}=9)$. Expression of all 4 genes was decreased in Piezo1 ${ }^{\triangle \mathrm{EC}}$ compared with Control. Normalization and Statistical analysis: mRNA expression was normalized to abundance of $\beta$-actin mRNA, which was not different between Control and Piezo ${ }^{\triangle \mathrm{EC}}$ (Supplementary Figure S3). t-Test was used, indicating significant difference of Piezo1 $^{\Delta \mathrm{EC}}$ cf Control $* \mathrm{P}<0.05, * * \mathrm{P}<0.01$.

\section{Discussion}

The study has identified a connection between Piezo1 channels and Notch1 signalling and thus a mechanism by which Notch1 regulation by force can be achieved. We propose a pathway in which activation of Piezo1 channels stimulates ADAM10 via $\mathrm{Ca}^{2+}$ and $\mathrm{Ca}^{2+}$-calmodulin-regulated mechanisms ${ }^{17,18}$ for S2 cleavage of Notch1, which then enables intracellular S3 cleavage by $\gamma$-secretase and release of NICD for association with transcriptional regulators such as RBPJ and the control of gene expression (Figure 5). In this way, Notch1 is coupled to an exceptional force sensor, the Piezo1 channel. Other mechanisms by which Notch1 achieves force sensitivity are not excluded but we suggest that Piezo1 is a mechanism of biological significance because endothelial-specific disruption of Piezo1 in vivo disturbed Notch1-regulated gene expression in hepatic endothelium, a site known to be Notchregulated. Our findings are consistent with prior studies of $D$ melanogaster that inferred Notch to be downstream of Piezo ${ }^{29}$ and so the proposed mechanism may have broad relevance. 


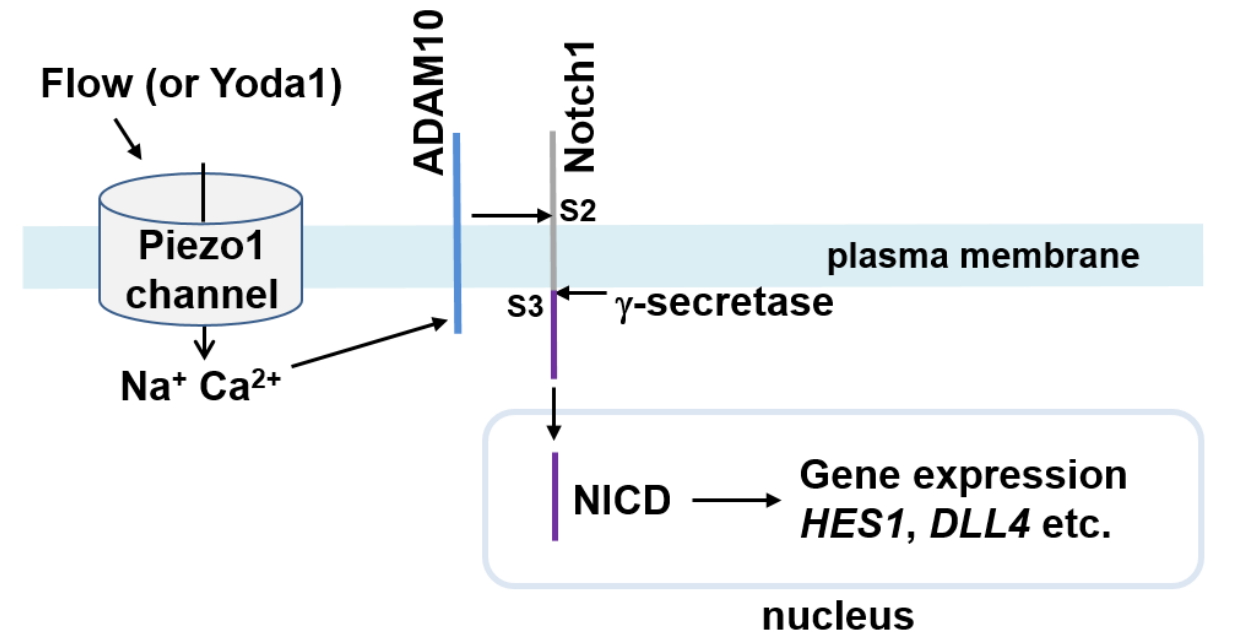

Figure 5: Summary of the proposed pathway. Activation of Piezol channel by mechanical force (e.g. fluid flow) or chemical agonist (Yoda1) causes elevation of the intracellular $\mathrm{Ca}^{2+}$ concentration which stimulates enzymatic activity of ADAM10 to cause S2 and S3 Notch1 cleavage and release of NICD to drive gene expression that is important in hepatic vasculature and potentially other vascular biology.

Prior work showed important effects of endothelial-specific disruption of Notch1-regulated RBPJ on hepatic microvasculature ${ }^{19,28}$. Disruption of RBPJ in 6 week-old mice led to enlarged sinusoids between portal and central venules, and disruption earlier in postnatal development caused poor perfusion, hypoxia and liver necrosis ${ }^{28}$. Therefore our observed requirement for Piezo1 in Notch1-regulated gene expression of hepatic endothelial cells suggests a positive role for the proposed Piezo1ADAM10/Notch1 partnership in hepatic function. It is important to emphasise that Piezo1 signalling is unlikely to be limited to ADAM10/Notch1 because there is also good evidence for Piezo1 coupling to calpain $^{9}, \operatorname{eNOS}^{9,14}$ and other mechanisms ${ }^{30}$. Therefore, it will be challenging to delineate the specific in vivo phenotypic contribution of Piezo1 coupling to ADAM10/Notch1 relative to other downstream consequences of activated Piezo1 channels.

An important observation of ours was that shear stress activates ADAM10 via Piezo1 (Figure 2h). It is consistent with previous work describing activation of ADAM10 by shear stress in human platelets ${ }^{31}$, which also express Piezo1 ${ }^{32}$. The finding has implications for Notch1 signalling but also more widely because ADAM10 is a sheddase that also targets E- and VE-cadherin, CD44 and cellular prion protein $^{17,33-35}$. Therefore its shear stress regulation via Piezo1 may be broadly relevant, for example in adherens junction biology and cartilage integrity, where functional importance of Piezo1 is already suggested $^{36,37}$, and amyloid plaque formation, where Piezo1 was originally detected ${ }^{38}$ and has been suggested to have functional importance in combination with $E$ coli infection ${ }^{39}$.

A prior report has suggested lack of specificity of Yoda1 for Piezo1 channels ${ }^{40}$ but the GsMTx4 toxin used as the basis of this proposal has limited value as a tool for evaluating Piezo1. Other prior studies have shown that genetic deletion of Piezo1 abolishes Yoda1's effects ${ }^{10,36,41,42}$ and that Piezo1 knockdown by RNA interference suppresses its effects ${ }^{14,30,43-46}$, consistent with Yoda1 having only Piezo1-mediated effects. Specific structure-activity requirements of Yoda1 at Piezo1 have been observed ${ }^{24}$ and it does not activate Piezo $2^{21}$. Here we observed that Piezo1-specific siRNA suppressed or abolished Yoda1 effects. Although it is important to seek Piezo1-specific activation with agents such as Yoda1 (because mechanical force is not specific to Piezo1) we recognise that activation by such agents does not necessarily mimic activation by a physiological factor such as shear stress. Importantly, therefore, we show that shear stress mimicked the effect of Yoda1 at ADAM10 and that genetic disruption of Piezo1 impacted Notch1 signalling in the absence of Yoda1. Therefore Piezo1 regulation of the ADAM10/Notch1 pathway was not dependent on Yoda1. 
In conclusion, we connect the new discovery of Piezo1 mechanosensing with the extensive prior discoveries of the ADAM and Notch fields ${ }^{1,19}$. The ability of Piezo1 to activate ADAM10 and Notch1 suggests that these mechanisms are downstream of Piezo1 and therefore linked via Piezo1 to changes in physiological force. There are likely to be broad-ranging implications for vascular and other biology. Understanding how to specifically disrupt the Piezo1-ADAM10/Notch1 partnership will be important as a next step to deciphering the roles and therapeutic potential of the pathway.

\section{Materials and Methods}

Piezo1 mutant mice. All animal use was authorized by the University of Leeds Animal Ethics Committee and Home Office UK. Genotypes were determined using real-time PCR with specific probes designed for each gene (Transnetyx, Cordova, TN). C57BL/6 J mice with Piezol gene flanked with LoxP sites $\left(\mathrm{Piezo}^{\text {flox }}\right)$ were described previously9. To generate tamoxifen (TAM) inducible disruption of Piezol gene in the endothelium (Piezol $\left.{ }^{\triangle E C}\right)$, Piezo ${ }^{\text {flox }}$ mice were crossed with mice expressing cre recombinase under the Cadherin5 promoter $(\mathrm{Tg}(\mathrm{Cdh} 5$-cre/ERT2)1Rha) and inbred to obtain Piezo $1^{\text {flox/flox} / C d h 5-c r e ~ m i c e . ~ T A M ~(T 5648, ~ S i g m a-A l d r i c h, ~ S a i n t-L o u i s, ~ M O) ~ w a s ~ d i s s o l v e d ~ i n ~ c o r n ~ o i l ~}$ (C8267 Sigma-Aldrich) at $20 \mathrm{mg} \cdot \mathrm{ml}^{-1}$. 10-12 week-old male mice were injected intra-peritoneal with $75 \mathrm{mg} \cdot \mathrm{kg}^{-1}$ TAM for 5 consecutive days and studied 10-14 days later. Control mice were the same except they lacked cre, so they could not disrupt Piezo1 even though they were also injected with TAM.

Acute isolation of liver endothelial cells. Liver of 12-14 week-old male mice was used. Tissue was mechanically separated using forceps, further cut in smaller pieces and incubated at $37{ }^{\circ} \mathrm{C}$ for $50 \mathrm{~min}$, in a MACSMix Tube Rotator to provide continuous agitation, along with $0.1 \%$ Collagenase II (17101015, Gibco, Waltham, MA) and Dispase Solution (17105-041, Gibco). Following enzymatic digestion samples were passed through $100 \mu \mathrm{m}$ and $40 \mu \mathrm{m}$ cell strainers to remove any undigested tissue. The suspension was incubated for $15 \mathrm{~min}$ with dead cell removal paramagnetic beads (130-090-101, Miltenyi Biotec GmbH, Bergisch Gladbach, Germany) and then passed through LS column (130-042401, Miltenyi Biotec). The cell suspension was incubated with CD146 magnetic beads (130-092-007, Miltenyi Biotec 130-092-007) at $4^{\circ} \mathrm{C}$ for 15 min under continuous agitation and passed through MS column (130-042-201, Miltenyi Biotec). The CD146 positive cells, retained in the MS column, were plunge out with PEB and centrifuged at 1000 RPM for $5 \mathrm{~min}$. Cell pellet was resuspended in RLT buffer (74004, Qiagen, Hilden, Germany) to proceed with RNA isolation.

Cell culture. HMVEC-Cs were cultured in endothelial medium 2MV (EGM-2MV, CC-3202, Lonza, Basel, Switzerland) according to the manufacturer's protocol. Sixteen hours before performing experiments, cells were cultured with starvation medium consisting of EGM-2MV but only $0.5 \%$ fetal bovine serum and without vascular endothelial growth factor $\mathrm{A}_{165}$ (VEGF $\mathrm{A}_{165}$ ) and basic fibroblast growth factor.

siRNA transfection. HMVEC-Cs were transfected with siRNA using Opti-MEM ${ }^{\mathrm{TM}}$ I Reduced Serum Medium (31985070, ThermoFisher Scientific, Waltham, MA) and Lipofectamine 2000 (11668019, ThermoFisher Scientific). For transfection of cells in 6-well plates, a total of $50 \mathrm{nmol}$ siRNA in $0.1 \mathrm{~mL}$ was added to $0.8 \mathrm{~mL}$ cell culture medium per well. Medium was changed after 4 hours. After 48 hours cells were treated or not with Yoda1 and subjected to RNA or protein isolation. For $\mathrm{Ca}^{2+}$ measurement, cells were plated into a 96-well plate at a density of 25000 cells per well 24 hours after transfection, and $\mathrm{Ca}^{2+}$ entry was recorded 24 hours later.

RNA isolation and RT-qPCR. For isolated liver endothelial cells, RNA was isolated by using RNeasy micro-kit (74004, Qiagen). A total of 100 ng RNA per sample was subjected to Reverse Transcriptase (RT) by using iScript ${ }^{\mathrm{TM}}$ cDNA Synthesis kit (1708890, BioRad, Hercules, CA). For whole liver, RNA was isolated using phenol/chloroform extraction from snap frozen samples. One $\mu \mathrm{g}$ of RNA was used for RT (Superscript® III Reverse Transcriptase, 18080044, Invitrogen, Carlsbad, CA). qPCR was performed using SyBR Green (1725122, Biorad). The sequences of PCR primers are shown in Supplementary Table S1. Primers were synthetized by Sigma. qPCR reactions were performed on a LightCycler® 480 Real Time PCR System (Roche, Basel, Switzerland). Samples were analysed using 
bioRxiv preprint doi: https://doi.org/10.1101/732370; this version posted August 12, 2019. The copyright holder for this preprint (which was not certified by peer review) is the author/funder, who has granted bioRxiv a license to display the preprint in perpetuity. It is made available under aCC-BY 4.0 International license.

the comparative CT method, where fold-change was calculated from the $\Delta \Delta \mathrm{Ct}$ values with the formula $2^{-\Delta \Delta C t}$.

ADAM10 enzyme activity. Activity was determined using the SensoLyte ${ }^{\circledR 520}$ ADAM10 Activity Assay Kit (AS-72226, AnaSpec Inc, Fremont, CA), which is based on the FRET substrate 5FAM/QXL ${ }^{\mathrm{TM}} 520$ with excitation/emission of $490 / 520 \mathrm{~nm}$. HMVEC-Cs were treated with or without Yoda1 for $30 \mathrm{~min}$ in the presence or absence of ADAM10 inhibitor. Cells were then washed with PBS and collected with Trypsin-EDTA. The pellet was resuspended in assay buffer, incubated on ice for $10 \mathrm{~min}$ and centrifuged at $10000 \mathrm{~g}$ for $10 \mathrm{~min}$ at $4^{\circ} \mathrm{C}$. The supernatants were plated on a 96-well plate. The substrate solution was diluted in Assay Buffer, brought to $37{ }^{\circ} \mathrm{C}$ was then mixed 1:1 with the sample. The fluorescence was measured every $2.5 \mathrm{~min}$ for $60 \mathrm{~min}$ at $37{ }^{\circ} \mathrm{C}$ at excitation/emission of 490/520 nm Flexstation 3 microplate reader with SoftMax Pro 5.4.5 software (Molecular Devices, San Josa, CA).

Shear stress. Endothelial cells were seeded on glass slides (MENSJ5800AMNZ, VWR, Radnor, PA) coated with Fibronectin (F0895, Sigma-Aldrich). Sixteen hours before performing experiments, cells were cultured with starvation medium consisting of EGM-2MV but only $0.5 \%$ fetal bovine serum and without vascular endothelial growth factor $\mathrm{A}_{165}$ (VEGF $\mathrm{A}_{165}$ ) and basic fibroblast growth factor. The slides were placed in a parallel flow chamber and flow of starvation medium was driven using a peristaltic pump.

Measurement of intracellular $\mathbf{C a}^{2+}$ concentration $\left(\left[\mathbf{C a}^{2+}\right]_{\mathrm{i}}\right)$. Cells plated in $96-$ well plates were incubated for 1 hour in Standard Bath Solution (SBS, containing in mM: $130 \mathrm{NaCl}, 5 \mathrm{KCl}, 8 \mathrm{D}$-glucose, 10 HEPES, $1.2 \mathrm{MgCl}_{2}, 1.5 \mathrm{CaCl}_{2}$, pH 7.4) supplemented with $2 \mu \mathrm{M}$ fura-2-AM (F1201, Molecular Probes, Eugene, OR) and $0.01 \%$ pluronic acid. Cells were then washed in SBS at room temperature for $30 \mathrm{~min}$, allowing deesterification to release free fura-2. Fluorescence $(\mathrm{F})$ acquisition (excitation 340 and $380 \mathrm{~nm}$; emission $510 \mathrm{~nm}$ ) was performed on a Flexstation 3 microplate reader with SoftMax Pro 5.4.5 software (Molecular Devices). After 60 seconds of recording, Yoda1 was injected. $\mathrm{Ca}^{2+}$ entry was quantified after normalization $(\Delta \mathrm{F} 340 / 380=\mathrm{F} 340 / 380(\mathrm{t})-\mathrm{F} 340 / 380(\mathrm{t}=0))$.

Immunoblotting. Proteins were isolated in RIPA buffer supplemented with PMSF, protease inhibitor mixture, and sodium orthovanadate (RIPA Lysis Buffer System, sc24948, Santa Cruz, Dallas, TX). Samples were heated at $95{ }^{\circ} \mathrm{C}$ for $5 \mathrm{~min}$ in SDS-PAGE sample buffer, loaded on a precast $4-20 \%$ polyacrylamide gradient gel (4561094, Biorad) and subjected to electrophoresis. Proteins were transferred onto a nitrocellulose membrane (Trans-Blot ${ }^{\circledR}$ Turbo $^{\mathrm{TM}}$ RTA Mini Nitrocellulose Transfer Kit, 1704270, BioRad) for 30 min using Trans-Blot Turbo Transfer System (BioRad). Membranes were blocked with $5 \%$ milk in Tris-buffered saline with Tween $0.05 \%$ for 1 hour at room temperature. The membranes were exposed to primary antibody overnight at $4{ }^{\circ} \mathrm{C}$, rinsed and incubated with appropriate horseradish peroxidase-labelled secondary antibody for 1 hour at room temperature. The detection was performed by using SuperSignal ${ }^{\mathrm{TM}}$ West Femto (34096, ThermoFisher Scientific) and visualized with a G-Box Chemi-XT4 (SynGene, Cambridge, UK). GAPDH was used as reference protein.

Reagents. Human cardiac microvascular endothelial cells (HMVEC-C, CC-7030, Lonza), DAPT (D5942, Sigma-Aldrich), GI254023X (3995, Tocris Bioscience, Bristol, UK), Yoda1 (5586/10, Tocris Bioscience), ON-TARGET plus Control siRNA (Dharmacon, Lafayette, CO), siRNA Piezo1 (SigmaAldrich: 5'- GCAAGUUCGUGCGCGGAUU[dT][dT]- 3'), ON-TARGET plus SMARTpool human siRNA ADAM10 (Dharmacon), cleaved Notch1 Val1744 D3B8 rabbit monoclonal (4147, Cell Signaling Technology, Danvers, MA), rabbit anti-ADAM10 (AB19026, Merck KGaA, Darmstadt, Germany), goat anti human VEGFR2 (AF357, R\&D system, Minneapolis, MN), mouse anti-human PECAM-1 (CD31) (M0823, Agilent Dako, Santa Clara, CA), GAPDH mouse anti-human (10R-G109b, Fitzgerald Industries International, Acton, MA) and anti-mouse, anti-rabbit and anti-goat HRP conjugated secondary antibodies (Jackson ImmunoResearch, Ely, UK). 
bioRxiv preprint doi: https://doi.org/10.1101/732370; this version posted August 12, 2019. The copyright holder for this preprint (which was not certified by peer review) is the author/funder, who has granted bioRxiv a license to display the preprint in perpetuity. It is made available under aCC-BY 4.0 International license.

Statistical Analysis. All averaged data are presented as mean \pm standard deviation (SD). Statistical significance was determined using two-tailed $t$-test when only two groups were compared or by 2-way ANOVA followed by Tukey posthoc test when multiple groups were treated with vehicle control (DMSO) or Yoda1 were studied. The genotypes of mice were blinded to the experimental investigator and studied at random according to Mendelian ratio. In all cases, statistical significance was assumed for probability $(P)<0.05$. Statistical tests were performed using OriginPro 8.6 software or GraphPad Prism 6.0. Outlying data were not detected or excluded. The letter $n$ indicates the number of independent biological experiments and its value in each case is stated in figure legends. The study was aimed at discovering components of a biological mechanism using various cell/molecular and animal-based studies to address a single hypothesis. In the absence of prior knowledge of the mechanism, power calculations were not considered to be applicable. We selected numbers of independent repeats of experiments base on prior experience of studies of this type. In all cases the number of independent repeats was at least 3 . The number of replicates per independent experiment was 1 for western blotting, 2 for $\mathrm{qPCR}, 4$ for $\mathrm{Ca}^{2+}$ assays and 1 for the ADAM10 activity assay.

\section{Supplementary Information}

Supplementary information is provided as a separate document. Included in this document are images of the uncropped western blots presented concisely in the main figures (Supplementary Figure S5).

\section{Acknowledgements}

The study was supported by EU Marie Skłodowska Curie Individual Fellowship to VC and by grants from the Wellcome Trust and British Heart Foundation to DJB and a Fonds Wetenschappelijk Onderzoek grant G091018N to EAVJ. We thank Fiona Bartoli for PCR primer design and Richard Cubbon for helpful comments on the manuscript.

\section{References}

1 Siebel, C. \& Lendahl, U. Notch Signaling in Development, Tissue Homeostasis, and Disease. Physiological reviews 97, 1235-1294, doi:10.1152/physrev.00005.2017 (2017).

2 Gordon, W. R. et al. Mechanical Allostery: Evidence for a Force Requirement in the Proteolytic Activation of Notch. Developmental cell 33, 729-736, doi:10.1016/j.devcel.2015.05.004 (2015).

3 Fang, J. S. et al. Shear-induced Notch-Cx37-p27 axis arrests endothelial cell cycle to enable arterial specification. Nature communications 8, 2149, doi:10.1038/s41467-017-01742-7 (2017).

4 Mack, J. J. et al. NOTCH1 is a mechanosensor in adult arteries. Nature communications $\mathbf{8}$, 1620, doi:10.1038/s41467-017-01741-8 (2017).

5 Lee, J. et al. 4-Dimensional light-sheet microscopy to elucidate shear stress modulation of cardiac trabeculation. The Journal of clinical investigation 126, 3158, doi:10.1172/JCI89549 (2016).

6 Jahnsen, E. D. et al. Notch1 is pan-endothelial at the onset of flow and regulated by flow. PloS one 10, e0122622, doi:10.1371/journal.pone.0122622 (2015).

7 Coste, B. et al. Piezo1 and Piezo2 are essential components of distinct mechanically activated cation channels. Science 330, 55-60, doi:10.1126/science.1193270 (2010).

8 Murthy, S. E., Dubin, A. E. \& Patapoutian, A. Piezos thrive under pressure: mechanically activated ion channels in health and disease. Nature reviews. Molecular cell biology 18, 771783, doi:10.1038/nrm.2017.92 (2017).

9 Li, J. et al. Piezo1 integration of vascular architecture with physiological force. Nature 515, 279-282, doi:10.1038/nature13701 (2014).

10 Rode, B. et al. Piezo1 channels sense whole body physical activity to reset cardiovascular homeostasis and enhance performance. Nature communications 8, 350, doi:10.1038/s41467017-00429-3 (2017). 
11 Ranade, S. S. et al. Piezo1, a mechanically activated ion channel, is required for vascular development in mice. Proceedings of the National Academy of Sciences of the United States of America 111, 10347-10352, doi:10.1073/pnas.1409233111 (2014).

12 Wu, J., Lewis, A. H. \& Grandl, J. Touch, Tension, and Transduction - The Function and Regulation of Piezo Ion Channels. Trends in biochemical sciences 42, 57-71, doi:10.1016/j.tibs.2016.09.004 (2017).

13 Maneshi, M. M., Ziegler, L., Sachs, F., Hua, S. Z. \& Gottlieb, P. A. Enantiomeric Abeta peptides inhibit the fluid shear stress response of PIEZO1. Scientific reports 8, 14267, doi:10.1038/s41598-018-32572-2 (2018).

14 Wang, S. et al. Endothelial cation channel PIEZO1 controls blood pressure by mediating flowinduced ATP release. The Journal of clinical investigation 126, 4527-4536, doi:10.1172/JCI87343 (2016).

15 Lewis, A. H. \& Grandl, J. Mechanical sensitivity of Piezo1 ion channels can be tuned by cellular membrane tension. eLife 4, doi:10.7554/eLife.12088 (2015).

16 Syeda, R. et al. Piezo1 Channels Are Inherently Mechanosensitive. Cell reports 17, 1739-1746, doi:10.1016/j.celrep.2016.10.033 (2016).

17 Nagano, O. et al. Cell-matrix interaction via CD44 is independently regulated by different metalloproteinases activated in response to extracellular $\mathrm{Ca}(2+)$ influx and $\mathrm{PKC}$ activation. The Journal of cell biology 165, 893-902, doi:10.1083/jcb.200310024 (2004).

18 Maretzky, T. et al. The cytoplasmic domain of a disintegrin and metalloproteinase 10 (ADAM10) regulates its constitutive activity but is dispensable for stimulated ADAM10dependent shedding. The Journal of biological chemistry 290, 7416-7425, doi:10.1074/jbc.M114.603753 (2015).

19 Alabi, R. O., Farber, G. \& Blobel, C. P. Intriguing Roles for Endothelial ADAM10/Notch Signaling in the Development of Organ-Specific Vascular Beds. Physiological reviews 98, 2025-2061, doi:10.1152/physrev.00029.2017 (2018).

20 Anders, A., Gilbert, S., Garten, W., Postina, R. \& Fahrenholz, F. Regulation of the alphasecretase ADAM10 by its prodomain and proprotein convertases. FASEB journal : official publication of the Federation of American Societies for Experimental Biology 15, 1837-1839 (2001).

21 Syeda, R. et al. Chemical activation of the mechanotransduction channel Piezo1. eLife 4, doi:10.7554/eLife.07369 (2015).

22 Lacroix, J. J., Botello-Smith, W. M. \& Luo, Y. Probing the gating mechanism of the mechanosensitive channel Piezo1 with the small molecule Yoda1. Nature communications $\mathbf{9}$, 2029, doi:10.1038/s41467-018-04405-3 (2018).

23 Wang, Y. et al. A lever-like transduction pathway for long-distance chemical- and mechanogating of the mechanosensitive Piezo1 channel. Nature communications 9, 1300, doi:10.1038/s41467-018-03570-9 (2018).

24 Evans, E. L. et al. Yoda1 analogue (Dooku1) which antagonizes Yoda1-evoked activation of Piezo1 and aortic relaxation. British journal of pharmacology 175, 1744-1759, doi:10.1111/bph.14188 (2018).

25 Imbimbo, B. P. Therapeutic potential of gamma-secretase inhibitors and modulators. Current topics in medicinal chemistry 8, 54-61 (2008).

26 Ludwig, A. et al. Metalloproteinase inhibitors for the disintegrin-like metalloproteinases ADAM10 and ADAM17 that differentially block constitutive and phorbol ester-inducible shedding of cell surface molecules. Combinatorial chemistry \& high throughput screening $\mathbf{8}$, 161-171 (2005).

27 Caolo, V. et al. Feed-forward signaling by membrane-bound ligand receptor circuit: the case of NOTCH DELTA-like 4 ligand in endothelial cells. The Journal of biological chemistry $\mathbf{2 8 5}$, 40681-40689, doi:10.1074/jbc.M110.176065 (2010).

28 Cuervo, H. et al. Endothelial notch signaling is essential to prevent hepatic vascular malformations in mice. Hepatology 64, 1302-1316, doi:10.1002/hep.28713 (2016).

29 He, L., Si, G., Huang, J., Samuel, A. D. T. \& Perrimon, N. Mechanical regulation of stem-cell differentiation by the stretch-activated Piezo channel. Nature 555, 103-106, doi:10.1038/nature25744 (2018). 
bioRxiv preprint doi: https://doi.org/10.1101/732370; this version posted August 12, 2019. The copyright holder for this preprint (which was not certified by peer review) is the author/funder, who has granted bioRxiv a license to display the preprint in perpetuity. It is made available under aCC-BY 4.0 International license.

30 Albarran-Juarez, J. et al. Piezo1 and Gq/G11 promote endothelial inflammation depending on flow pattern and integrin activation. The Journal of experimental medicine 215, 2655-2672, doi:10.1084/jem.20180483 (2018).

31 Facey, A. et al. A-Disintegrin-And-Metalloproteinase (ADAM) 10 Activity on Resting and Activated Platelets. Biochemistry 55, 1187-1194, doi:10.1021/acs.biochem.5b01102 (2016).

32 Ilkan, Z. et al. Evidence for shear-mediated $\mathrm{Ca}(2+)$ entry through mechanosensitive cation channels in human platelets and a megakaryocytic cell line. The Journal of biological chemistry 292, 9204-9217, doi:10.1074/jbc.M116.766196 (2017).

33 Maretzky, T. et al. ADAM10 mediates E-cadherin shedding and regulates epithelial cell-cell adhesion, migration, and beta-catenin translocation. Proceedings of the National Academy of Sciences of the United States of America 102, 9182-9187, doi:10.1073/pnas.0500918102 (2005).

34 Schulz, B. et al. ADAM10 regulates endothelial permeability and T-Cell transmigration by proteolysis of vascular endothelial cadherin. Circulation research 102, 1192-1201, doi:10.1161/CIRCRESAHA.107.169805 (2008).

35 Jarosz-Griffiths, H. H. et al. Proteolytic shedding of the prion protein via activation of metallopeptidase ADAM10 reduces cellular binding and toxicity of amyloid-beta oligomers. The Journal of biological chemistry 294, 7085-7097, doi:10.1074/jbc.RA118.005364 (2019).

36 Friedrich, E. E. et al. Endothelial cell Piezo1 mediates pressure-induced lung vascular hyperpermeability via disruption of adherens junctions. Proceedings of the National Academy of Sciences of the United States of America, doi:10.1073/pnas.1902165116 (2019).

37 Lee, W. et al. Synergy between Piezo1 and Piezo2 channels confers high-strain mechanosensitivity to articular cartilage. Proceedings of the National Academy of Sciences of the United States of America 111, E5114-5122, doi:10.1073/pnas.1414298111 (2014).

38 Satoh, K. et al. A novel membrane protein, encoded by the gene covering KIAA0233, is transcriptionally induced in senile plaque-associated astrocytes. Brain research 1108, 19-27, doi:10.1016/j.brainres.2006.06.050 (2006).

39 Velasco-Estevez, M. et al. Infection Augments Expression of Mechanosensing Piezo1 Channels in Amyloid Plaque-Reactive Astrocytes. Frontiers in aging neuroscience 10, 332, doi:10.3389/fnagi.2018.00332 (2018).

40 Dela Paz, N. G. \& Frangos, J. A. Yoda1-induced phosphorylation of Akt and ERK1/2 does not require Piezo1 activation. Biochemical and biophysical research communications 497, 220225, doi:10.1016/j.bbrc.2018.02.058 (2018).

41 Cahalan, S. M. et al. Piezo1 links mechanical forces to red blood cell volume. eLife 4, doi:10.7554/eLife.07370 (2015).

42 Romac, J. M., Shahid, R. A., Swain, S. M., Vigna, S. R. \& Liddle, R. A. Piezo1 is a mechanically activated ion channel and mediates pressure induced pancreatitis. Nature communications 9, 1715, doi:10.1038/s41467-018-04194-9 (2018).

43 Nonomura, K. et al. Mechanically activated ion channel PIEZO1 is required for lymphatic valve formation. Proceedings of the National Academy of Sciences of the United States of America 115, 12817-12822, doi:10.1073/pnas.1817070115 (2018).

44 Iring, A. et al. Shear stress-induced endothelial adrenomedullin signaling regulates vascular tone and blood pressure. The Journal of clinical investigation 130, 2775-2791, doi:10.1172/JCI123825 (2019).

45 Tsuchiya, M. et al. Cell surface flip-flop of phosphatidylserine is critical for PIEZO1-mediated myotube formation. Nature communications 9, 2049, doi:10.1038/s41467-018-04436-w (2018).

46 Liu, C. S. C. et al. Cutting Edge: Piezo1 Mechanosensors Optimize Human T Cell Activation. Journal of immunology 200, 1255-1260, doi:10.4049/jimmunol.1701118 (2018). 
Supplementary Information

Supplementary Table S1. PCR primer sequences.

\begin{tabular}{llll}
\hline Species & Gene & Forward & Reverse \\
\hline $\begin{array}{llll}\text { Homo } \\
\text { Sapiens }\end{array}$ & GAPDH & GCCTCAAGATCATCAGCAAT & GGACTGTGGTCATGAGTCCT \\
& DLL4 & ACAACTTGTCGGACTTCCAG & CAGCTCCTTCTTCTGGTTTG \\
& HES1 & CCAAAGACAGCATCTGAGCA & GCCGCGAGCTATCTTTCTT \\
& PIEZO1 & CGTCTTCGTGGAGCAGATG & GCCCTTGACGGTGCATAC \\
& ADAM10 & TTGCCTCCTCCTAAACCACTTCCA & AGGCAGTAGGAAGAACCAAGGCAA \\
\hline $\begin{array}{l}\text { Mus } \\
\text { Musculus }\end{array}$ & D-actin & CCAGATCTTCTCCATGTCGT & CCAGATCTTCTCCATGTCGT \\
& All4 & ACTTCGTCTGCAACTGTCCT & CAGCACCAGCAGTACCACTA \\
& CCTCTGAGCACAGAAAGTCA & GCCGGGAGCTATCTTTCTTA \\
& Heyl & GTACCCAGTGCCTTTGAGAA & TTTCAGGTGATCCACAGTCA \\
& EphrinB2 & GGCCTGGTACTATACCCACA & ATTGTGCATCTGTCTGCTTG \\
& Piezol & TGAGCCCTTCCCCAACAATAC & CTGCAGGTGGTTCTGGATATAG \\
& Tie2 & AAGCAACCCAGCCTTTTCTC & TGAGCATTCTCCTTTGGAC \\
\hline
\end{tabular}


a.

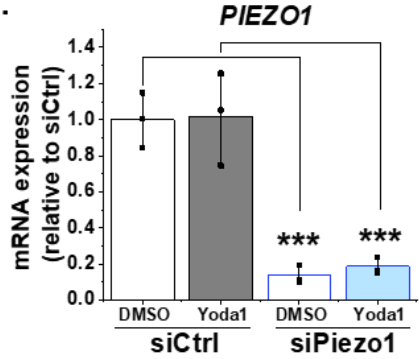

e.

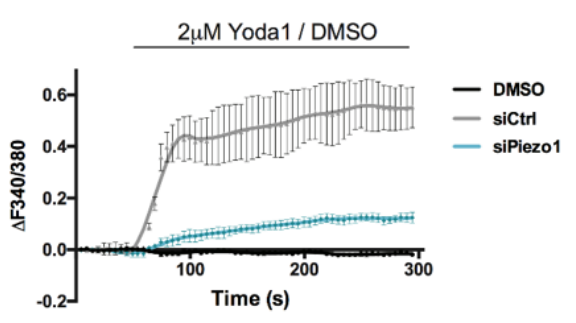

b.

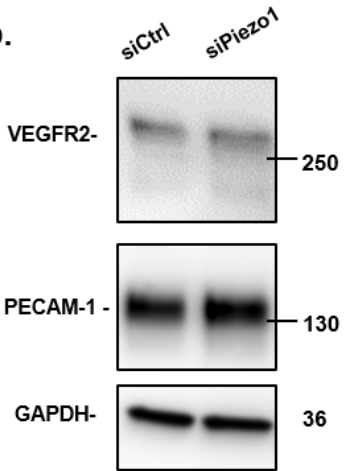

f.

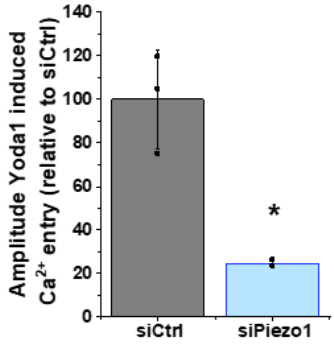

c.

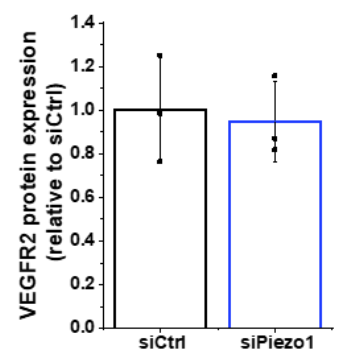

d.

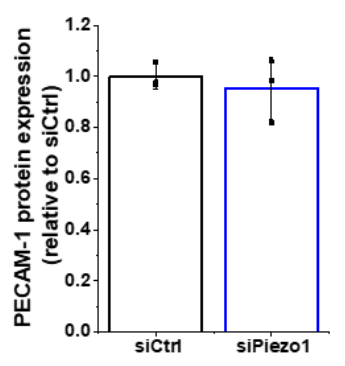

g.

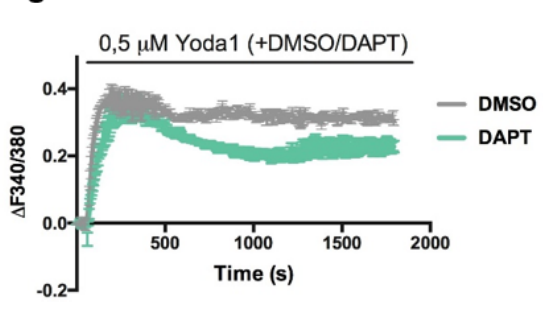

h.

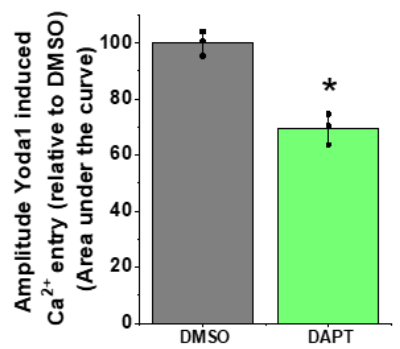

Supplementary Figure S1: Supporting data for main Figure 1. (a) Summarized mean \pm SD $(n=3)$ quantitative PCR data for fold-change in Piezo1 mRNA in HMVEC-Cs treated for 30 min with $0.2 \mu \mathrm{M}$ Yoda1 or vehicle (DMSO) after transfection with control siRNA (siCtrl) or Piezo1 siRNA (siPiezo1). (b) Representative Western blot labelled with anti-VEGRF2, antiPECAM-1 (anti-CD31) and anti-GAPDH antibodies for HMVEC-Cs after transfection with control siRNA (siCtrl) or Piezo1 siRNA (siPiezo1). (c, d) Quantification of data of the type exemplified in (b), showing mean \pm SD data for abundance of VEGFR2 (c) and PECAM-1 (d) normalized to $\operatorname{siCtrl}(\mathrm{n}=3)$. (e-f) Representative intracellular $\mathrm{Ca}^{2+}$ measurement traces (e) in HMVEC-Cs during application of $2 \mu \mathrm{M}$ Yoda1 or its control DMSO, 48 hours after transfection with control siRNA (siCtrl) or Piezo1 siRNA (siPiezo1), shown as mean \pm SD of the amplitude for $n=3(\mathbf{f})$. (g-h) Representative intracellular $\mathrm{Ca}^{2+}$ measurement traces (g) in HMVEC-Cs during prolonged application of $0.5 \mu \mathrm{M}$ Yodal or its vehicle control (DMSO) simultaneously with $10 \mu \mathrm{M}$ DAPT or its vehicle control (DMSO), shown as mean \pm SD of the area under the curve for $n=3$ (h). Two-way ANOVA test was used for (a) and t-test for $(\mathbf{c}, \mathbf{d}, \mathbf{f}, \mathbf{h})$, indicating $* \mathrm{P}<0.05, * * * \mathrm{P}<0.001$. 
bioRxiv preprint doi: https://doi.org/10.1101/732370; this version posted August 12, 2019. The copyright holder for this preprint (which was not certified by peer review) is the author/funder, who has granted bioRxiv a license to display the preprint in perpetuity. It is made available under aCC-BY 4.0 International license.

a.

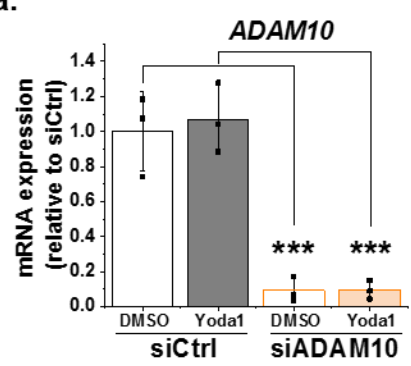

b.

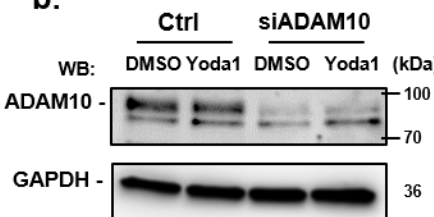

c.

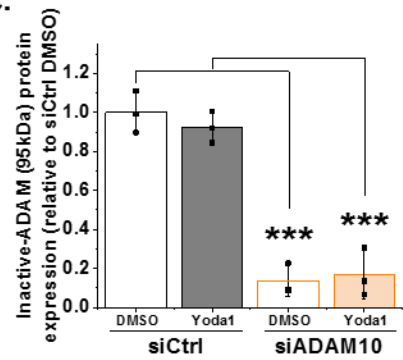

d.

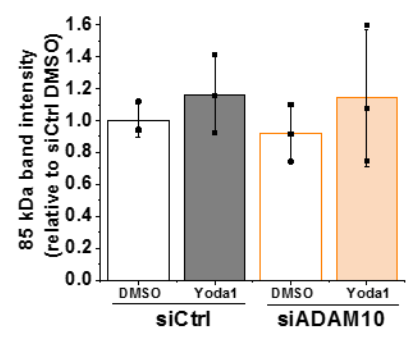

e.

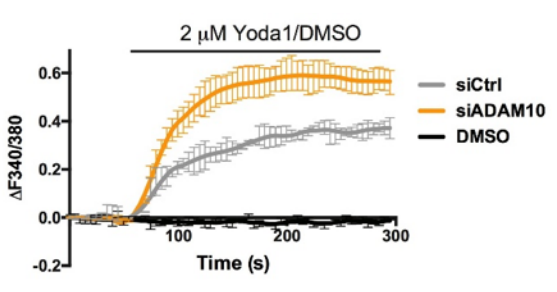

i.

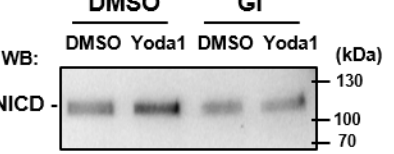

f.

j.
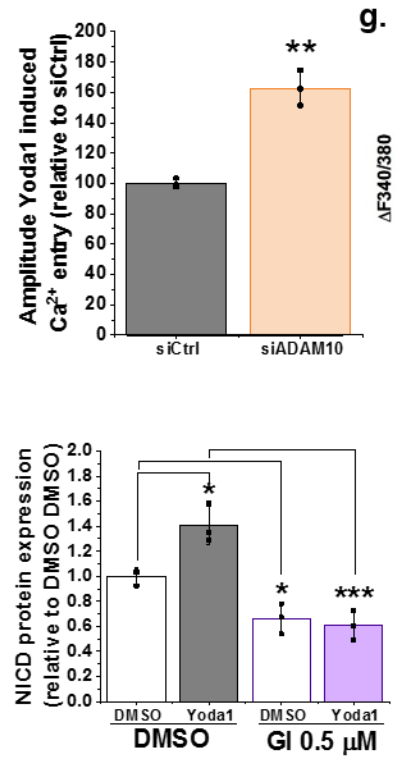

g.

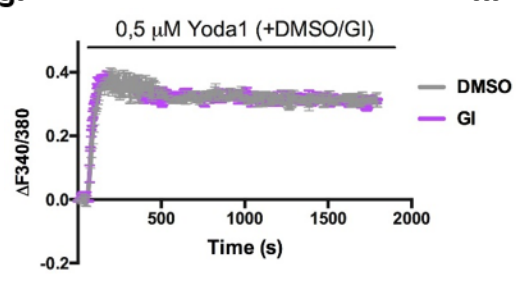

k.

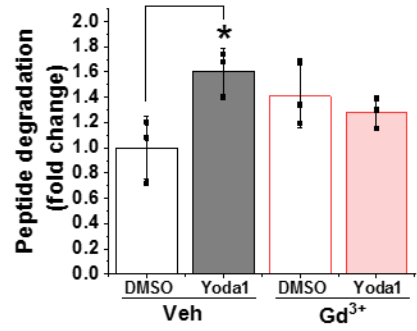

Supplementary Figure S2: Supporting data for main Figure 2. (a) Summarized mean \pm SD $(n=3)$ quantitative PCR data for fold-change in ADAM10 mRNA in HMVEC-Cs treated for 30 min with $0.2 \mu$ M Yoda1 or vehicle (DMSO) after transfection with control siRNA (siCtrl) or ADAM10 siRNA (siADAM10). (b) Representative Western blot labelled with anti-ADAM10 antibody for HMVEC-Cs after transfection with control siRNA (siCtrl) or ADAM10 siRNA (siADAM10). (c, d) Quantification of data of the type exemplified in (b), showing mean \pm SD data for abundance of uncleaved ADAM10 (95 $\mathrm{kDa})(\mathbf{c})$ and non-specific labelling of an unknown protein $(85 \mathrm{kDa})(\mathbf{d})$ normalized to siCtrl $(\mathrm{n}=3)$. (e-f) Representative intracellular $\mathrm{Ca}^{2+}$ measurement traces (e) in HMVEC-Cs during application of $2 \mu \mathrm{M}$ Yoda1 or its vehicle control (DMSO), 48 hours after transfection with control siRNA (siCtrl) or ADAM10 siRNA (siADAM10), shown as mean \pm SD of the amplitude for $\mathrm{n}=3(\mathbf{f})$. (g-h) Representative intracellular $\mathrm{Ca}^{2+}$ measurement traces $(\mathbf{g})$ in HMVEC-Cs during prolonged application of $0.5 \mu \mathrm{M}$ Yodal or its vehicle control (DMSO) simultaneously with $5 \mu \mathrm{M}$ GI254023X (GI) or its vehicle control (DMSO), shown as mean \pm SD of the area under the curve for $n=3(\mathbf{h})$. (i) Representative Western blot labelled with anti-NICD and anti-GAPDH antibodies for HMVEC-Cs treated for $30 \mathrm{~min}$ with $0.2 \mu \mathrm{M}$ Yoda1 or vehicle (DMSO) in the absence or presence of $0.5 \mu \mathrm{M}$ GI254023X (GI) (j) Quantification of data of the type exemplified in (i), showing mean \pm SD data for abundance of NICD normalized to siCtrl DMSO $(n=3)$. (k) ADAM10 enzyme activity assessed by specific peptide degradation and subsequent fluorescence emission after 30 min treatment of HMVEC-Cs with $0.2 \mu \mathrm{M}$ Yoda1 in the absence or presence of 30 $\mu \mathrm{M} \mathrm{Gd}^{3+}$. Data are shown as mean \pm SD data $(\mathrm{n}=3)$ relative to vehicle condition. Statistical analysis: Two-way ANOVA test was used, indicating $* \mathrm{P}<0.05, * * \mathrm{P}<0.01$ and $* * * \mathrm{P}<0.001$ 
a.

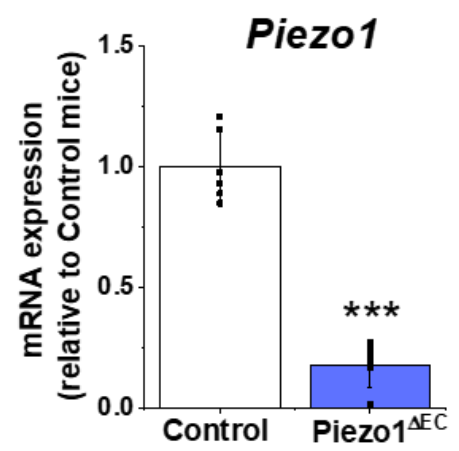

b.

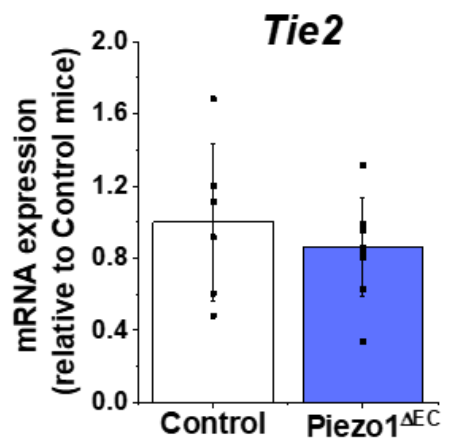

C.

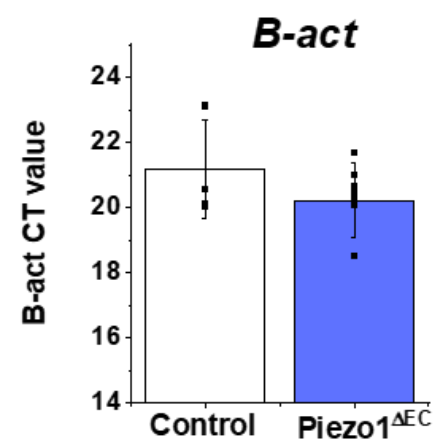

Supplementary Figure S3: Supporting data for main Figure 4. Additional gene quantification for liver endothelial cells from Piezo1 ${ }^{\triangle \mathrm{EC}}$ and Control mice. (a) Piezo1. (b) Tie2. (c) $\beta$-actin (raw CT values). (a, b) Normalized to abundance of $\beta$-actin (reference) expression. Statistical analysis: $\mathrm{t}$-test was used for comparisons, $* * * \mathrm{P}<0.001$.

a.

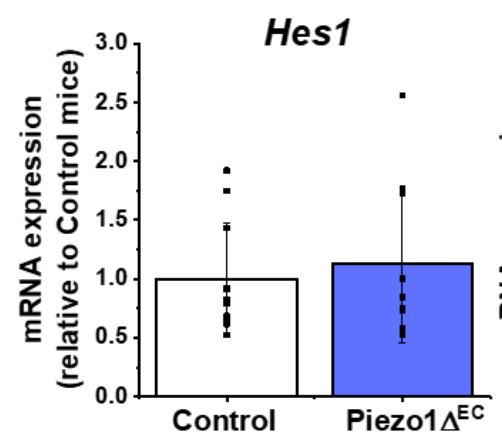

b.

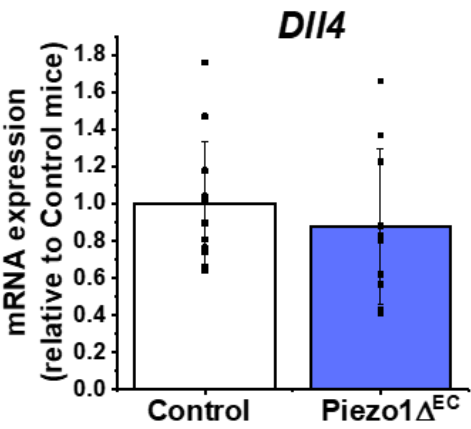

c.

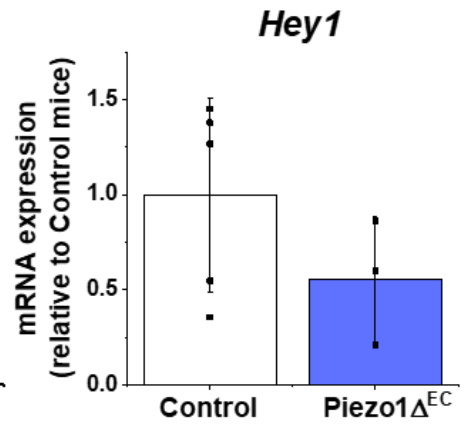

d.

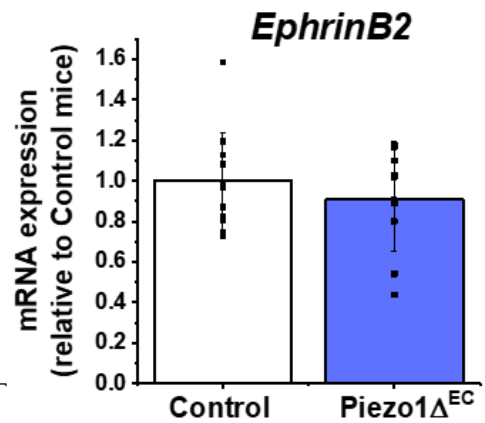

Supplementary Figure S4: Notch1-regulated gene expression in whole liver. Gene quantification for whole liver from Piezo1 $^{\triangle \mathrm{EC}}(\mathrm{n}=10)$ and Control mice $(\mathrm{n}=12)$. (a) Hesl. (b) Dll4. (c) Heyl (in 7 Control and 7 Piezo1 ${ }^{\triangle \mathrm{EC}}$ experiments Heyl expression was not convincingly detected, so these data were excluded). (d) Ephrin B2. Normalized to abundance of the reference $\beta$-actin expression. Statistical analysis: t-test was used for comparisons. There were no significant differences between any pairs. 
bioRxiv preprint doi: https://doi. org/10.1101/732370; this version posted August 12, 2019. The copyright holder for this preprint (which was not certified by peer review) is the author/funder, who has granted bioRxiv a license to display the preprint in perpetuity. It is made available under aCC-BY 4.0 International license.

Fig. $1 a$

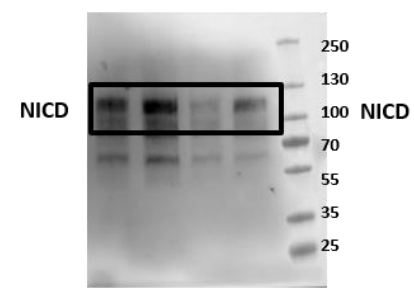

GAPDH

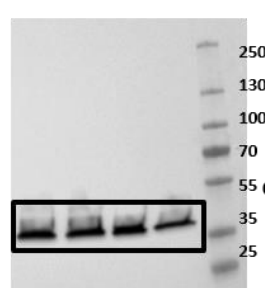
55 GAPD$$
35
$$

Fig. 1c

\section{Suppl. Fig. 1b}

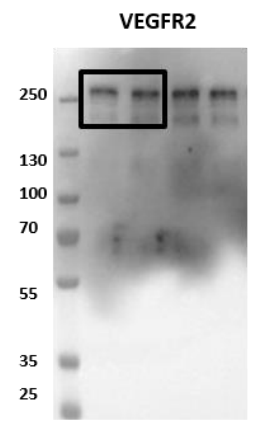

Fig. 2a

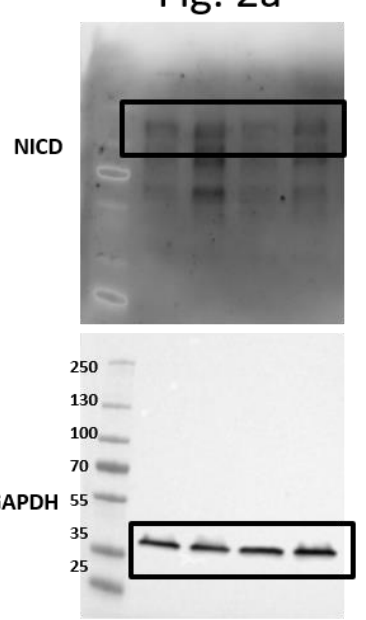

Fig. 2c

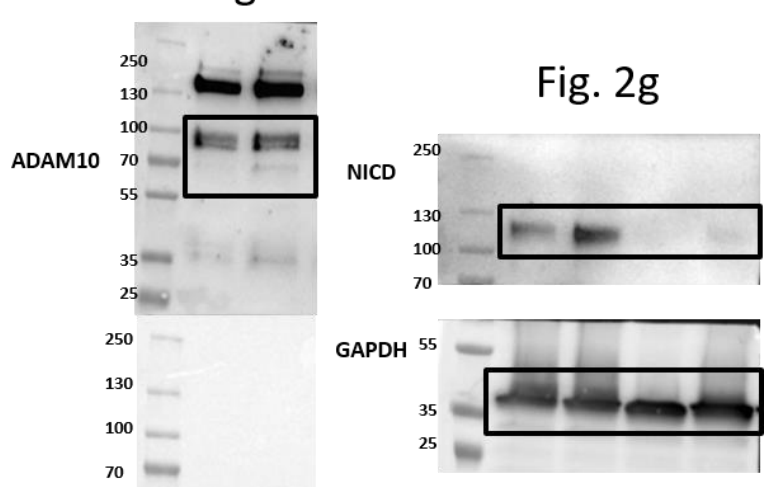

GAPDH ${ }^{55}$

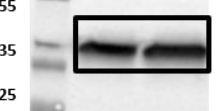

25

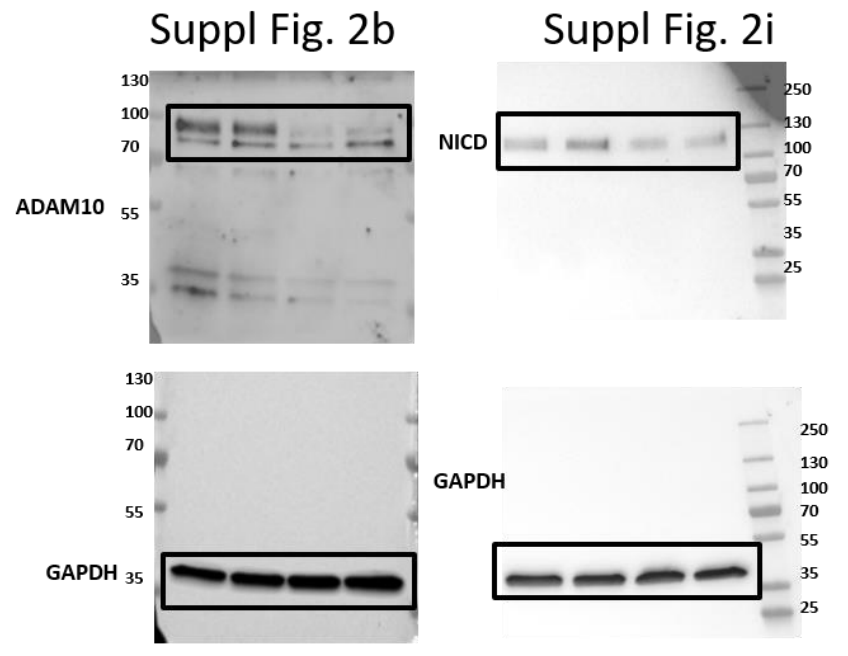

Supplementary Figure S5: Uncropped western blots. 